for example, lead and thallium; the following 38 pages being devoted to microvolumetric analysis.

The Strohlein or Metrohm plunger-type burettes with 'Teflon' pistons and a capacity of $2 \mathrm{ml}$. are recommended for N/50 titrants. Gorbach burettes are described, but the presence of a meniscus causes after-flow. The British 'Agla' syringe can be used both as a pipette and as a burette.

Much attention is directed to ethylenediamine tetraacetic acid microtitrations, and there are numerous practical examples of direct replacement and of back titrations using $0.02 \mathrm{M}$ solutions.

The volume ends with a most useful chapter on quantitative elementary organic analysis. Carbon and hydrogen are determined by the method of Belcher and Godbert; the halogens by the ter Meulen-Sloof technique or elternatively by the Schoniger oxygen-flask. Nitrogen is determined by ter Meulen hydrogenation using $\mathrm{Ni}^{-\mathrm{ThO}_{2}}$ catalyst or by the modified Dumas method. Didactic considerations have led Van Nieuwenburg and Van Ligten to exclude descriptions of well-known methods such as those of Unterzaucher for oxygen or Zimmermann for sulphur.

A useful description is, however, given of molecular weight determination on a micro-scale utilizing the 'isothermal distillation' technique as suggested by Barger, developed by Wright and modified by Van Nieuwenburg and Van Ligten.

This publication has a wholesome practical bias and will have an undoubted value and appeal to teachers of microanalysis in academic institutions. It has also much to offer the practising anajyst who may be called on to work with rather restricted quantities of a parent substance or who wishes to economize on his financial outlay.

D. T. Lewrs

\section{FOREIGN CHEMICALS IN FOODS}

\section{Residue Reviews}

Residues of Pesticides and other Foreign Chemicals in Foods and Feeds. Edited by Francis A. Gunther. Vol. 1. Pp.iv + 162. Vol. 2. Pp. iv +156 . Vol. 3. Pp. iv +170 . (Berlin: Springer-Verlag, 1963.) 22 D.M. each volume.

$\mathrm{T}$ HE incorporation, often inadvertently, of foreign substances in foodstuffs has caused increasing concern in recent years. Attention has been directed especially to residues of chemicals which are needed to control pests, diseases and weeds in crops in the field, to combat parasites of animals used for food, or to protect foodstuffs in transit or storage. Much work has been devoted to finding how the crop protection and related chemicals may be safely used. A new and specialized field of residue analysis has emerged allowing the levels of contamination in harvested crops to be determined, and the behaviour and ultimate fate of the chemicals have been extensively investigated. Some are lost from crops by weathering, some are degraded into harmless derivatives by the plant or animal, and others are eliminated during food processing. Some chemicals, however, persist on crops and in soil, tend to accumulate in animal food chains, and may endanger public health in due course. Associated with the chemical examinations are toxicological investigations to determine the significance of the residues that may be found. Statutory and other measures adopted to protect the consumer include the institution of permissible limits of contamination, maximum recommended rates of use of potentially harmful chemicals, and the observance of adequate periods for weathering.

The object of Residue Reviews is to bring together the many aspects of the problem of foreign chemicals in foodstuffs. The use of substances to improve flavour, appearance, etc., and contamination incurred during manufacture, packaging, etc., are also considered to be suitable subjects for discussion. Contributions are made by invitation, and an advisory board of specialists from many countries indicates the international scope of the publication.

The three volumes that have so far appeared deal largely with problems arising from the use of chemicals for crop protection. General articles describe the nature of plant surfaces in relation to the use of spray chemicals, the absorption, translocation and metabolism of chemicals in plants, and the processes involved in deposition, degradation, persistence and effectiveness. A valuable series of papers on analytical methods show the advantages conferred on residue analysis by modern techniques of chromatography, polarography and spectrophotometry. A few contributions discuss the levels of residues that are found in practice, one deals with the principles underlying the establishment of residue tolerances, and another examines the difficulties and interpretation of tests for carcinogenicity. Each article gives summaries in English, French and German and useful references.

Work on residues in foodstuffs is expanding so rapidly, and information is scattered in so many journals, that undoubtedly there is ample soope for a publication of this kind. To be fully effective, its professed aim of international coverage should be attained; at present emphasis rests on work and reports from the United States, a position that no doubt will change when the series becomes established. Many of the contributions give valuable information, but some scarcely reach the standard that may reasonably be expected. A considerable amount of tidying-up is needed before the presentation will satisfy critical appraisal: proprietary and coined names of chemicals are used indiscriminately, inconsistencies occur in chemical names, and the subject index is quite inadequate. This last deficiency detracts much from the merits of an otherwise commendable, if expensive, publication.

J. T. Martin

\section{ANALYSING FOOD CONTAMINANTS AT LENGTH}

Analytical Methods for Pesticides, Plant Growth Regulators and Food Additives

Edited by Gunter Zweig. Vol. 1: Principles, Methods, and General Applications. Pp. xiv + 637. (Now York: Academic Press, Inc.; London: Academic Press, Inc. (London), Ltd., 1963.) 171s. 6d.

DARKINSON'S Law states that work will always expand to fill the time available for its execution. The same principle surely applies to publishers with an infinite supply of paper, editors with an inexhaustible number of contributors and libraries with a seemingly unlimited provision of dollars. The present book is priced at $£ 811 s$. $6 d$. Its purpose is to serve only as an introductory review of the principles and general applications of the analytical methods which are to be described in Volumes 2, 3, 4 and 5 that have yet to be published. The fortunate possessor of the entire compilation, who will, if he reads all the 3,185 pages which it will doubtless comprise, know all there is to know about the analysis of crops and food for traces of pesticides, plant growth regulators and food additives, will therefore have paid $£ 4217 s$. $6 d$. for the privilege.

A number of the authors of individual chapters discuss what they call the "clean-up procedures" by which they concentrate the trace residues in which they are interested and free them from the bulk of the sample. If an analogous process could have been applied to the book itself, everyone would have been the gainer because there is much in it of value. W. Thornburg, for example, contributes an admirable, short, clear and practical chapter on the various methods by which trace contaminants of different classes can be separated from the materials with which an 